\title{
Carência de cálcio e vitamina $D$ em crianças e adolescentes: uma realidade nacional
}

Natasha Aparecida Grande de França ${ }^{1}$; Bárbara Santarosa Emo Peters ${ }^{2}$; Lígia Araújo Martini ${ }^{3}$

\section{Resumo}

O cálcio e a vitamina D são nutrientes essenciais para a saúde óssea, e o adequado fornecimento destes implica em melhor formação do esqueleto e, consequentemente, em adequado crescimento infantil e prevenção da ocorrência de osteoporose na vida adulta. Há, porém, evidências de elevada prevalência de inadequação de ingestão destes nutrientes entre a população de crianças e adolescente brasileiros, o que reflete mudanças comportamentais e de hábitos dietéticos e tem como consequência principal os possíveis impactos sobre a saúde óssea ao decorrer da vida.

Palavras chave: deficiência de vitamina D; deficiência de cálcio; infância; adolescência

De França, Natasha Aparecida Grande; Peters, Bárbara Santarosa Emo; Martini, Lígia Araújo. "Carência de cálcio e vitamina D em crianças e adolescentes: uma realidade nacional", in Anais do 20. Congresso Internacional Sabará de Especialidades Pediátricas

\footnotetext{
${ }^{1}$ Mestra em Nutrição em Saúde Pública pela Faculdade de Saúde Pública da Universidade de São Paulo (FSP/USP).natasha.agf@gmail.com

2 Pós-doutoranda no Departamento de Nutrição da Faculdade de Saúde Pública da Universidade de São Paulo (FSP/USP). bsepeters@hotmail.com

${ }^{3}$ Prof $^{a}$ Livre Docente e Associada nível III do Departamento de Nutrição da Faculdade de Saúde Pública da Universidade de São Paulo (FSP/USP). Imartini@usp.br
} 


\section{Introdução}

Este artigo traz dados atuais sobre a prevalência de inadequação de ingestão de cálcio e vitamina D entre crianças e adolescentes brasileiros e discute sobre a importância da investigação, prevenção e tratamento da deficiência destes micronutrientes.

O cálcio e a vitamina D são nutrientes essenciais para a saúde óssea, e o adequado fornecimento dos mesmos, desde o período gestacional (pela gestante) até o final da puberdade, implica em melhor formação do esqueleto e consequentemente em adequado crescimento infantil e prevenção da ocorrência de osteoporose na vida adulta (Zhu e Prince, 2012). Estudos apontam que o aumento em um desvio padrão (DP) no pico de massa óssea (ápice da formação do osso, que ocorre geralmente entre os 18 e 20 anos) pode reduzir em até 50\% o risco de fraturas ao longo da vida (Boot et al., 2010; Baxter-Jones et al., 2011).

Aproximadamente 99\% do cálcio encontra-se no osso, onde juntamente com o fósforo formam os sais de hidroxiapatita $\left(\mathrm{Ca}_{5}\left(\mathrm{PO}_{4}\right)_{3} \mathrm{OH}\right)$, garantindo a dureza e função de sustentação característica do esqueleto (Bonucci, 2012). Entretanto, para que a homeostase do cálcio ocorra de forma adequada, faz-se necessária a ingestão combinada com concentrações séricas suficientes de vitamina D, uma vez que esta colabora positivamente no processo de absorção do cálcio no lúmen intestinal, bem como na reabsorção de cálcio no osso, garantindo que a remodelação óssea ocorra dentro dos parâmetros de normalidade (DeLuca, 2004).

\section{2. $O$ cálcio}

O cálcio é um mineral fundamental para a manutenção de inúmeras funções no organismo. Além da saúde óssea, ele também é necessário aos processos de coagulação sanguínea, transmissão nervosa, função neuromuscular e permeabilidade das membranas celulares (Joint Health Claims Initiative to the Food Standards Agency, 2003). Dessa forma o Brasil adota as recomendações de ingestão preconizadas pelo Instituto de Medicina (Institute of Medicine, 2011), as quais variam de acordo com a faixa etária, sendo menores no início da infância (700 mg/dia), e maiores na puberdade (1.300 $\mathrm{mg}$ /dia), justamente por se tratar do período que antecede o pico de massa óssea. É importante enfatizar, entretanto, que são aceitáveis valores 200 mg inferiores (Estimated Average Requirements EAR), mas é fundamental garantir a máxima adequação à recomedação (Recommended Dietary Allowances - RDA).

A principal fonte alimentar de cálcio é o leite e seus derivados (queijos, iogurte), não somente por apresentarem boas quantidades nas porções usualmente consumidas, mas também pelo fato de se tratar das fontes em que o cálcio é mais biodisponível, possivelmente pela presença da lactose, a qual por agir como prebiótico no intestino grosso elevaria a solubulidade do cálcio (Pereira et al., 2009). Além disso, uma vez que as recomendações de cálcio são elevadas, o consumo dos latícinios pode ser 
traduzido como o meio mais simples de alcança-las, isto porque outras fontes, como os vegetais verde escuro, além de apresentarem menor biodisponibilidade não são usualmente bem aceitos pelo paladar info-juvenil.

Dessa forma a baixa ingestão de alimentos lácteos na infância e adolescência pode ser traduzida em maior risco de fraturas. Kalkwarf et al. (2003) encontraram um aumento em 60\% na ocorrência de fraturas ao longo da vida quando ingestão menor ou igual à uma porção de leite por semana entre crianças e adolescentes (OR: 1,60; 95\%IC: 1,17 - 2,18). Entretanto, os resultados da Pesquisa de Orçamentos Familiares 2008 - 2009 revelaram que os adolescentes brasileiros estão ingerindo cada vez mais bebidas açucaradas, como sucos artificiais e refrigerantes, em relação (e provavelmente em substituição) ao leite, iogurte e bebidas lácteas.

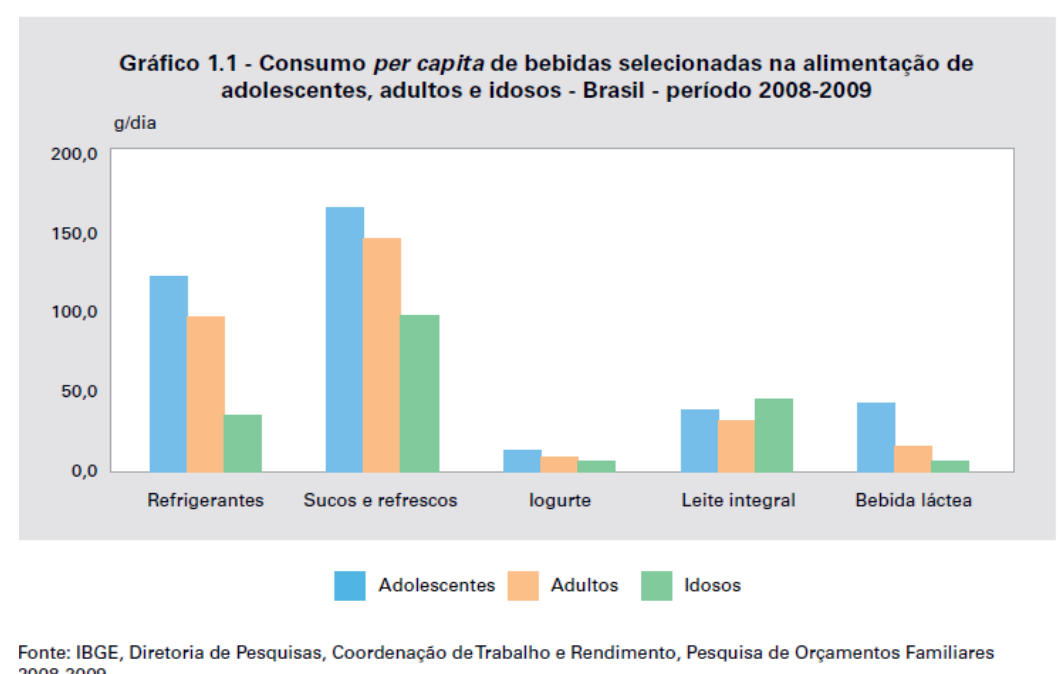

Figura 1: Pesquisa de Orçamentos Familiares 2008 - 2009

E, de fato a baixa ingestão desses alimentos reflete em baixa ingestão de cálcio. A própria POF revelou uma média de ingestão de 535 mg de cálcio entre jovens de 10 a 18 anos de idade, ou seja, aproximandamente 52\% de inadequação em relação à recomendação. Entre residentes no município de São Paulo, o estudo ISA-Capital (Martini et al., 2013) encontrou resultados ainda mais preocupantes ao avaliar adolescentes de ambos os sexos, os quais apresentaram 93\% de ingestão inadequada de cálcio. Entre crianças, o Estudo Nutri-Brasil Infância (Bueno et al., 2013) avaliou a ingestão de 3.058 crianças de 2 a 6 anos de escolas públicas e privadas e encontrou uma média de prevalência de inadequação de $45 \%$ entre aquelas na faixa etária de 4 a 6 anos, sem diferença entre as que frequentavam o ensino público ou privado, o que indica que os efeitos das mudanças nos hábitos alimentares nacionais estende-se às diferentes classes sociais.

Os resultados desses estudos revelam a crescente prevalência de deficiência de ingestão de cálcio no Brasil entre as crianças e adolescentes, o que provavelmente resultará em consequências negativas para a saúde óssea dessa geração, além de maiores gastos para os sistemas de saúde. Com 
isso, faz-se fundamental que políticas públicas sejam traçadas a fim de incentivar a ingestão desses alimentos, principalmante durante essa fase de vida, a fim de reduzir os possíveis danos futuros ào nossa população.

\section{A vitamina D}

Concentrações séricas adequadas de vitamina D (VD) são fundamentais para a melhor absorção do cálcio dietético, as quais, segundo a Sociedade de Endocrinologia, são traduzidas em valores de vitamina D $\geq 75 \mathrm{nmol} / \mathrm{L}$ ( $\geq 30 \mathrm{ng} / \mathrm{mL}$ ) (Holick et al., 2011).

Devido sua associação com o metabolismo ósseo, a deficiência de VD pode resultar em distúrbios de crescimento durante a infância e, quando acentuada, em raquitismo. Entretanto, além de sua ação sobre o esqueleto, estudos tem mostrado que sua deficiência nessa fase de vida também estaria associada ao maior risco de infecções do trato respiratório (Dawodu, Nath, 2011), doenças autoimunes, como a Diabetes mellitus tipo 1(Hypponen et al., 2001), e posterior desenvolvimento de esclerose múltipla (Willer et al., 2005) e doença de Crohn (Bartels et al., 2013). Isto porque a VD é considerada um hormônio pleiotrópico, com capacidade de modulação da resposta do sistema imune inato (Liu et al., 2006) .

A VD é encontrada naturalmente em duas formas, o ergocalciferol (vitamina D2) produzido pelas plantas, e o colecalciferol (vitamina D3), sintetizado pelo tecido cutâneo animal mediante exposição à luz solar. Apesar de ainda controversas, as recomendações atuais de VD são de $10 \mu \mathrm{g}$ (400 UI) por dia, à partir do nascimento até os 8 anos, e de 15 ㅆ (600 UI) até o final da vida (Maeda et al., 2014).

O Estudo Nutri-Brasil Infância encontrou uma inadequação de aproximadamente $90 \%$ na ingestão de VD entre todas as crianças avaliadas (2 a 6 anos) (Bueno et al., 2013). Resultados que parecem se estender à população de adolescentes, uma vez que, tanto o estudo conduzido por Peters et al. (2009), quanto o estudo ISA-Capital (apenas no município de São Paulo) (Martini et al., 2013), demonstraram haver uma baixa ingestão dessa vitamina. Contudo, as fontes dietéticas de VD são poucas e os alimentos que apresentam boas concentrações não são consumidos habitualmente por nossa população, como é o caso do salmão (36 ㅆg em 155 g) (Garcia e Martini, 2010). Além disso, há no mercado nacional, poucos alimentos fortificados disponíveis, com isso, torna-se claro que a principal forma de obtenção de VD é por meio da exposição à radiação ultravioleta, a qual é capaz de ativar o 7-dehidrocolesterol presente na pele, desencadeando uma cascata de ativação e duas hidroxilações da VD, até que seja obtida sua forma ativa, capaz de realizar as funções a que se destina, o calcitriol [1,25(OH) $\left.)_{2} \mathrm{D}\right]$ (Maeda et al., 2014).

Entretanto, a irradiação solar sofre alterações sazonais (menor nos meses que contemplam o outono e o inverno) e também ao longo do dia (maior pico em torno do meio dia) (Maeda et al., 2013) que poderia implicar em variações quanto à produção de VD. Além disso, com o aumento da 
criminalidade e também nos casos de câncer de pele, somados à vida moderna, os responsáveis pelas crianças e adolescentes tem limitado sua exposição à ambientes externos e, consequentemente, à luz solar. Fator que pode ser agravado pelo sedentarismo e obesidade infanto-juvenil, uma vez que o excesso de tecido adiposo também é fator de risco para a deficiência de VD. Apesar de escassos, os estudos nacionais apontam para uma mesma direção. Peters et al. (2009) encontraram prevalência de insuficiência de VD em 62\% de 136 adolescentes moradores do interior de São Paulo. Oliveira et al. (2013) revelaram que aproximadamente 71\% dos adolescentes avaliados em Juiz de Fora - MG apresentavam VD sérica insuficiente. Resultados ainda mais alarmantes foram encontrados por e Santos et al. (2012), com 54,3\% de insuficiência e 36,3\% de deficiência entre crianças e adolescente de Curitiba - PR.

Para prevenção deste quadro, a Sociedade Americana de Pediatria, preconiza a suplementação com 400 UI (10 $\mu \mathrm{g}$ ) entre as crianças com amamentação exclusiva (quando a impossibilidade de adequada exposição solar), entre as crianças alimentadas com fórmulas fortificadas, mas que não ingerem quantidades suficientes, ou quando ingestão ou exposição solar insuficientes pelos adolescentes (Wagner e Greer, 2008). Entretanto, questiona-se quanto à eficácia dessa dosagem, uma vez que parece ser suficiente para garantir uma não deficiência, mas não necessariamente um estado de suficiência (Gallo et al., 2013) e também quanto à suplementação indiscriminada, uma vez que sugere-se que somente as crianças com deficiência comprovada seriam de fato beneficiadas (Winzenberg et al., 2011).

Portanto, há a necessidade de que mais estudos sejam conduzidos, principalmente longitudinais, a fim de se avaliar o real impacto da suplementação e suas consequências na vida futura dessas crianças, e também de mais discussões sobre o impacto dessa suplementação sobre a amamentação exclusiva (o leite materno não era suficiente?).

\section{CONCLUSÃO}

Há entre a população de crianças e adolescente brasileiros, uma elevada prevalência de inadequação de ingestão de cálcio e vitamina D (esta também pela insuficiente exposição solar), o que reflete mudanças comportamentais e de hábitos dietéticos e tem como consequência principal os possíveis impactos sobre a saúde óssea na vida adulta, aumentando o risco de desenvolvimento de osteoporose e fraturas. 
Calcium and vitamin D deficiency in children and adolescents: a national reality

Abstract: Calcium and vitamin D are essential nutrients for bone health. The adequate intake of both implies in a more adequate skeleton formation and, consequently, contributes positively to child growth and to prevention of osteoporosis in adulthood. There is, however, evidence of high prevalence of inadequate intake of these nutrients in the population of Brazilian children and adolescents, which reflects changes in behavioral and dietary habits, leading to possible negative impacts on bone health in later life.

Keywords: Vitamin D deficiency; Calcium deficiency; Childhood; Adolescence

\section{Referencias}

Bartels LE, Jorgensen SP, Bendix M, Hvas CL, Agnholt J, Agger R, et al. 25-Hydroxy vitamin D3 modulates dendritic cell phenotype and function in Crohn's disease. Inflammopharmacology. 2013;21(2):177-86.

Baxter-Jones ADG, Faulkner RA, Forwood MR, Mirwald RL, Bailey DA. Bone mineral accrual from 8 to 30 years of age: an estimation of peak bone mass. J Bone Miner Res; 2011; 26: 17291739.

Bonucci E. Bone mineralization. Front Biosci (Landmark Ed). 2012;17:100-28.

Boot AM, Ridder MAJ, van der Sluis IM, van Slobbe, Krenning EP, de Muinck Keizer-Schrama SMPF. Peak bone mineral density, lean body mass and fractures. Bone; 2010; 46: 336-341.

Bueno MB, Fisberg RM, Maximino P, Rodrigues GP, Fisberg M. Nutritional risk among Brazilian children 2 to 6 years old: A multicenter study. Nutrition; 2013; 29: 405-410.

Dawodu A, Nath R. High prevalence of moderately severe vitamin D deficiency in preterm infants. Pediatr Int. 2011;53(2):207-10.

DeLuca HF. Overview of general physiologic features and functions of vitamin D. Am J Clin Nutr. 2004;80(6 Suppl):1689S-96S.

Gallo S, Comeau K, Vanstone C, Agellon S, Sharma A, Jones G, L’Abbe'M, Khamessan A, Rodd C, Weiler H. Effect of Different Dosages of Oral Vitamin D Supplementation on Vitamin D Status in Healthy, Breastfed Infants. A Randomized Trial. JAMA. 2013; 309(17):1785-1792.

Garcia VC, Martini LA. Vitamin D and Cardiovascular Disease. Nutrients. Apr 2010; 2(4): 426437.

Holick MF, Binkley NC, Bischoff-Ferrari HA, Gordon CM, Hanley DA, Heaney RP, et al. Evaluation, treatment, and prevention of vitamin D deficiency: an Endocrine Society clinical practice guideline. J Clin Endocrinol Metab. 2011;96(7):1911-30.

Hypponen E, Laara E, Reunanen A, Jarvelin MR, Virtanen SM. Intake of vitamin D and risk of type 1 diabetes: a birth-cohort study. Lancet. 2001;358(9292):1500-3.

Institute of Medicine. Dietary Reference Intakes for Calcium and Vitamin D. Washington, DC: The National Academies Press; 2011. 
Instituto Brasileiro de Geografi a e Estatística - IBGE. Pesquisa de orçamentos familiares 20082009: análise do consumo alimentar pessoal no Brasil. Rio de Janeiro: IBGE; 2011.

Joint Health Claims Initiative to the Food Standards Agency. Final Technical Report; 2003; pp 4-266. Kalkwarf HJ, Khoury JC, Lanphear BP. Milk intake during childhood and adolescence, adult bone density, and osteoporotic fractures in US women. Am J Clin Nutr 2003;77:257-65.

Liu PT, Stenger S, Li H, Wenzel L, Tan BH, Krutzik SR, et al. Toll-like receptor triggering of a vitamin D-mediated human antimicrobial response. Science. 2006;311(5768):1770-3.

Maeda SS, Borba VZC, Camargo MBR, Silva DMW, Borges JLC, Bandeira F, Lazaretti-Castro M. Recommendations of the Brazilian Society of Endocrinology and Metabology (SBEM) for the diagnosis and treatment of hypovitaminosis D. Endocrinol Metab. 2014;58(5):411-33.

Maeda SS, Saraiva GL, Hayashi LF, Cendoroglo MS, Ramos LR, Corrêa MP, Mesquita CH, Lazaretti-Castro M. Seasonal variation in the sérum 25-hydroxyvitamin $D$ levels of young and elderly active and inactive adults in São Paulo, Brazil. Dermato-Endocrinology. 2013; 5:1, 211217.

Martini LA, Verly Jr. E, Marchioni DML, Fisberg RM. Prevalence and correlates of calcium and vitamin D status adequacy in adolescents, adults, and elderly from the Health Survey - São Paulo. Nutrition; 2013; 29: 845-850.

Oliveira RM, Novaes JF, Azeredo LM, Candido AP, Leite IC. Association of vitamin D insufficiency with adiposity and metabolic disorders in Brazilian adolescents. Public Health Nutr. 2013; 9:1-8.

Pereira GAP, Genaro OS, Pinheiro MM, Szejnfeld VL, Martini LA. Cálcio dietético - estratégias para otimizar o consumo. Rev Bras Reumatol; 2009; 49: 164-80.

Peters BSE, Santos LC, Fisberg M, Wood RJ, Martini LA. Prevalence of Vitamin D Insufficiency in Brazilian Adolescents. Ann Nutr Metab 2009; 54:15-21.

Santos BR, Mascarenhas LP, Satler F, Boguszewski MC, Spritzer PM. Vitamin D deficiency in girls from South Brazil: a cross-sectional study on prevalence and association with vitamin $D$ receptor gene variants. BMC Pediatr. 2012;12:62.

Wagner CL, Greer FR. Prevention of Rickets and Vitamin D Deficiency in Infants, Children, and Adolescents. Pediatrics. 2008;122;1142

Willer CJ, Dyment DA, Sadovnick AD, Rothwell PM, Murray TJ, Ebers GC. Timing of birth and risk of multiple sclerosis: population based study. BMJ. 2005;330(7483):120.

Winzenberg T, Powell S, Shaw KA, Jones G. Effects of vitamin D supplementation on bone density in healthy children: systematic review and meta-analysis. BMJ 2011;342:c7254. Zhu K, Prince RL. Calcium and bone. Clin Biochem; 2012; 45: 936-942. 